\title{
Cell Biology in Space: Basic research and potential applications
}

\author{
A. Cogoli
}

Zero-g LifeTec GmbH, Zurich

The purpose of this paper is to present an overview of our work conducted in space and on ground in simulated microgravity the past three decades. When, 1976, I proposed to the European Agency to study the effect of weightlessness on cell differentiation, very few scientists believed that the absence of gravity might have an influence on the biological processes taking place in the microcosmos of single cells. Indeed, the differences between the density of cell organelles and that of the cytoplasm together with the flexibility of the cytoskeleton strongly support the notion that gravity may directly interfere with cellular processes.

As model for our studies we have selected the mitogenic activation in vitro of T cells. Such model permits to investigate crucial events occurring during the cellular immune response as well as a differentiation process in which resting $T$ lymphocytes are triggered to produce lymphokines (in particular interferon-gamma, interleukin-2 and its receptor) and to proliferate. Our interest focused both on the immune system of humans in space and on signal transduction in differentiating cells.

\section{Experiment with $T$ cells}

In November 1983 the Spacelab module was flown in the payload bay of the shuttle Columbia in the Spacelab I mission. In an incubator manufactured in our workshop we determined the mitotic index of $T$ cells activated and chemically fixed in flight. Activation of cells was determined by measuring the tritiated thymidine incorporated into DNA.There was a dramatic loss of activity (more than 95\%) compared to identical samples kept on the ground (Cogoli et al., 1984). That discovery triggered the interest of the scientific community and several experiments were performed by us and others in space and on ground in order to clarify how gravity could interfere with the signal transduction and differentiation mechanisms in T cells. In all following studies in space we used a reference centrifuge generating artificial I g gravity.

The results from Spacelab I were confirmed in 1985 in the Spacelab DI mission.

A new approach was adopted in a study performed in 1991 in Spacelab SLSI (there had been a long interruption of the shuttle flights after the catastrophe of Challenger in 1986).
T lymphocytes (known to be adhesion-independent cells) were coated to microcarrier beads. To our great surprise the depression of mitogenic activation observed in resuspended cells was not observed in coated cells (Bechler et al., 1992). On the same mission, by means of a "multi-g centrifuge" we observed that there must be a thresholf of sensitivity between 0 and $0.6 \mathrm{~g}$ (Cogoli et al., 1993).

In the last 20 years we have performed in collaboration with other laboratories (in Sassari, Rome, Florence, L'Aquila, S. Francisco and Berlin) several experiments in Spacelab, sounding rockets, and stratospheric balloons. Such experiments were accompanied by extensive investigations conducted in the ground laboratory in fast rotating bi- and three-dimensional clinostats.

In the Spacelab IML 2 mission, flown in 1994, a centrifuge microscope developed by the German Space Agency, DARA GmbH, based on plans proposed by Briegleb and Hemmersbach (Friedrich et al., 1996) permitted to record the movements and interactions of lymphocytes in microgravity. The experiment addressed a crucial question related to the loss of $T$ cell activation in space, namely whether the cells are capable of autonomous movements and of establishing cell-cell contacts under $0 \mathrm{~g}$ conditions. In fact, T cell activation requires tight contacts between each other as well between $T$ cells and monocytes as antigen-presenting cells. We were able to see that cells display autonomous movements and interactions in $0 \mathrm{~g}$ (Cogoli-Greuter et al., 1996).We investigated the structure of the cytoskeleton of the intermediate filaments of vimentin in Jurkat cells (a cell line derived from human $T$ cells) by immunofluorescence technique on the sounding rocket MAXUS IB.There was a significant higher formation of large bundles of filaments already $30 \mathrm{~s}$ after exposure to $0 \mathrm{~g}$ thus showing that the cytoskeleton undergoes important and immediate changes in microgravity (CogoliGreuter et al., 1998).

The structure of F-actin filaments was investigated in 2006 in a facility called KUBIK on board of the International Space Station, ISS. Again important differences between the distribution of actin between $\mathrm{I} g$ and $\mathrm{Og}$ in the macrophage cell line J-I I I were observed (Cogoli-Greuter et al., manuscript in preparation).

The data obtained in space as well as in clinostats can be summarized as follows: (i) already $\mathbf{3 0}$ seconds after 
exposure to microgravity there are significant changes in the structure of the cytoskeleton; (ii) cell-cell interactions and cell movements are taking place also in a weightless environment; (iii) the genetic expression of important proteins and factors involved in $T$ cell activation is significantly depressed; (iv) protein kinase A (PKA), in addition to protein kinase $C$ (PKC) and mitogen-activated protein kinase (MAPK) pathways, plays a role in early $T$ cell activation and induction of interleukin-2 (IL-2), interleukin2 receptor alpha subunit (IL-2R $\alpha$ ) and interferon-gamma (IFN- $\gamma$ ) gene expression (Hughes-Fulford et al., 2005); (v) the data show that the downregulation of early genes regulated primarily by transcription factors NF-K $B, C R E B$, ELK and STAT contribute to $T$ cell dysfunction in a microgravity environment; (vi) the fact that phosphorylation of the linker of activation in T cells (LAT) is not down-regulated in simulated microgravity indicates that cholesterol-rich lipid rafts are not involved in the down-regulation of the transcription factors (Boonyaratanakornkit et al., 2005), (vii) T cells undergo apoptosis in $0 \mathrm{~g}$ conditions (Maccarrone et al., 2003). In parallel with the investigations on signal transduction we have also studied the activation of T cells from astronauts prior to, in and post flight. The data confirm early findings of US and Russian scientists who reported a depression of the mitogenic activation in lymphocytes in blood samples taken immediately after flight. The values return to the baseline within one week after landing. It is still not clear whether such depression is caused by the exposure of the human body to weightlessness or rather to the physical and psychological stress of the space flight. The study of the impact on the immune system of astronauts that has been rather neglected in the past is gaining now high priority in the perspective of long duration missions to the planet Mars. A comprehensive review of our work with cells of the immune system has been published recently, (Cogoli and Cogoli-Greuter 2005).

\section{Potential applications}

The interesting and somehow unexpected behaviour of cells in space induced us to develop sophisticated instruments for biotechnological processes in space laboratory.A first step was the development ob a bioreactor that was flown on three space missions (Spacelab IML-2, STS-76 in 1996 and STS-107, that ended with the catastrophe of Columbia in 2003) with yeast cells of Saccharomyces cerevisiae. The analysis of the electron micrograph from the experiment on IML-2 showed a significant difference in the distribution of the bud scars (remaining after the separation of a daughter cell) between $\mathrm{I} g$ in flight controls and $0 \mathrm{~g}$ samples: while a majority of the scars show bipolar distribution at $\mathrm{I} g$, there is a significant higher random distribution at $0 \mathrm{~g}$. Walther et al. (1996). These data suggest that there are remarked changes in the intracellular structure.

In an effort to take profit of microgravity for biomedical and biotechnological applications we started a series of investigations conducted in the three-dimensional clinostat, called also random positioning machine, RPM.

One project is dedicated to the study of osteoporosis by means of a newly developed bone resorption assay: The experimental model consisted of the human bone marrow derived FLG 29.1 cell line, previously characterized as an osteoclastic precursor model which adhered to bone slices on which a rut was scraped with a scalpel. The profile of the rut was measured after 3 days of incubation in the RPM.There was a clear and measurable resorption of the bone under simulated $0 \mathrm{~g}$ conditions, whereas no change was detected in the control samples (Monici et al., 2006). We plan to use such model to tests drugs and food supplements that could prevent osteoporosis.

We studied the behaviour of epithelial cells in microgravity for two reasons. One is to better understand how wound healing in space proceeds. In fact, it is known since the early time of human space flight that wound healing is retarded in $0 \mathrm{~g}$. The other reason is to investigate angiogenesis in microgravity in order to explore whether the production of artificial tissue (e.g. blood vessels) could be improved in zero $\mathrm{g}$. The formation of a tube-like structure by epithelial cells was observed in the RPM (Infanger et al., 2006).A tubular structure (like a lumen) with a length of $1.8 \mathrm{~mm}$ and an artificial endothelium layer along the extracellular matrix were formed after $72 \mathrm{~h}$ culture under simulated microgravity.

Spheroids of tumour cells are useful cell aggregates that can be regarded as a tumour model suitable for the study of anti-tumoral drugs. We have investigated the formation and properties of spheroids of thyroid carcinoma cells grown in the RPM (Grimm et al., 2002).

In conclusion, these findings suggest that the alterations of single cell behavior observed in the absence of gravity may be exploited for biotechnological and biomedical applications. Examples from our own research in clinostats are (i) the study of osteoporosis in an in vitro model based on bone slices and osteoclasts; (ii) the formation of multicellular tumor spheroids as a model of solid tumors; (iii) the formation of cartilage tissue from chondrocytes.

\section{Acknowledgments}

The work of the author and collaborators described in this paper has been supported by the Swiss National Research Foundation, by the ETH Zurich, by the PRODEX Program of ESA, by NASA and by ASI. In addition the Author wishes to thank the teams of the ETH Zurich, of the Universities of Sassari, Florence and L'Aquila, of the Free University of Berlin and of the University of California, S. Francisco.The visiting professorship has been supported by the University of Sassari.

\section{References}

Bechler B., Cogoli A., Cogoli-Greuter M., Müller O., Hunzinger E. \& Criswell SB. 1992. Activation of microcarrier-attached lymphocytes in microgravity. Biotech. \& Bioeng., 40: $991-996$

Boonyaratanakornkit JB., Cogoli A., Chai-Fei Li. Schopper T., Pippia 
P., Galleri G., Meloni MA., Hughes-Fulford M., 2005. Title: Key gravity-sensitive signaling pathways drive T-cell activation. FASEB J., 19: 2020-2022.

Cogoli A. 1993. The effect of hypogravity and hypergravity on cells of the immune system. J. Leukoc. Biol., 54: 259-268.

Cogoli A., Bechler B., Cogoli-Greuter M., Criswell SB., joller H., Joller P., Hunzinger E. \& Müller O. 1993. Mitogenic signal transduction in T-lymphocytes in microgravity. J. Leukoc. Biol., 53: 569-575.

Cogoli A., Cogoli-Greuter M. 2007. Cells of the immune system in space (lymphocytes). In: Biology in space and life on Earth, Brinckmann E ed.,Wiley-VHC GmbH \& Co. KGaA : 193-222.

Cogoli A.,Tschopp A. \& Fuchs-Bislin P. 1984. Cell sensitivity to gravity. Science, 225: 228-230.

Cogoli-Greuter M., Meloni MA., Sciola L., Spano A., Pippia P., Monaco G. \& Cogoli A. 1996. Movements and interactions of leukocytes in microgravity. J. Biotechnol., 47: 279-287.

Cogoli-Greuter M., Spano A., Sciola L., Pippia P., Cogoli A. 1998. Influence of microgravity on mitogen binding, motility and cytoskeleton patterns of T lymphocytes and Jurkat cells. Experiments on sounding rockets. J. J.Aerospace Env. Med., 35: 27-39.

Friedrich ULD., Joop O., Pütz Ch., Willich G. 1996. The slow rotating centrifuge microscope NIZEMI - a versatile instrument for terrestrial hypergravity and space microgravity research in biology and material science. J. Biotechnol., 47: 225-238.

Grimm D., Bauer J., Kossmehl P., Shakibaei M., Schönberger ]., Pickenhahn H., Schulze-Tanzil G., Vetter R., Paul M., Cogoli A.
2002. Simulated microgravity alters differentiation and increases apoptosis in human follicular thyroid carcinoma cells. FASEB J., 16:604-606, published on line February 25, 2002.

Hughes-Fulford, Sugano E., Schopper T., Li C-F., Boonyratanakornkit JB., Cogoli A. 2005. Early immune response and regulation of IL-2 receptor subunits. Cellular signalling, $17: 1|1|-1 \mid 24$

Infanger M., Kossmehl, Shakibaei M., Baatout S.,Witzing A., Grosse J,Bauer J., Cogoli A., Faramarzi S., Derradji H., Neefs M.,.Paul M.,Grimm D. 2006. Induction of three-dimensional assembly and increase in apoptosis of human endothelial cells by simulated microgravity: impact of vascular endothelial factor. Apoptosis , 11: 749-764.

Maccarrone M., Battista N., Meloni MA., Bari M., Galleri G., Pippia P., Cogoli A., Finazzi-Agrò A. 2003. Creating conditions similar to those occurring during exposure of cells to microgravity induces apoptosis in human lymphocytes by 5 -lipoxigenasemediated mitochondrial uncoupling and cytochrome $c$ release. J. Leukoc. Biol., 73: 472-481.

Monici M., Fusi F., Paglierani M., Marziliano N., Cogoli A., Pratesi R., Bernabei PA. 2006. Modeled gravitational unloading triggers differentiation and apoptosis in preosteaoclastic cells. J. Cell. Biochem., 98: 65-80. [Epub: Dec 19]

Walther I., Bechler B., Müller O., Hunzinger E. \& Cogoli A. 1996. Cultivation of Saccharomyces cerevisiae in a bioreactor in microgravity. J. Biotechnol., 47: I 13-127. 